\title{
GLAUCONITA COMO FONTE ALTERNATIVA DE POTÁSSIO PARA PRODUÇÃO DE FERTILIZANTE DE LIBERAÇÃO LENTA
}

\author{
Antônio C. Pereira ${ }^{1}$, Marta R. S. Gomes ${ }^{2}$, Sônia D. F. Rocha ${ }^{1}$ \\ 1 Departamento de Engenharia Química da Universidade Federal de Minas Gerais - UFMG - Belo Horizonte - MG - Rua Guanabara, 491 - Concórdia. 31110 - \\ 650 Belo Horizonte MG. (claretipereira@gmail.com; Autor correspondente). \\ 2 Secretaria de Estado de Desenvolvimento Econômico, Ciência, Tecnologia e Ensino Superior de MG - SEDECTES. Prédio Gerais - Rodovia Prefeito Américo \\ Renê Gianetti, 4001 - Serra Verde, Belo Horizonte - MG, 31630-901
}

Recebido em 1 de março de 2019, aceito em 22 dezembro de 2019

Resumo: A glauconita $\left(\mathrm{K}_{2}(\mathrm{MgFe})_{2} \mathrm{Al}_{6}\left(\mathrm{Si}_{4} \mathrm{O}_{10}\right)_{3}(\mathrm{OH})_{12}\right)$ é um mineral monoclínico, de cor verde, filossilicato hidratado de potássio e ferro. É uma fonte alternativa de potássio. Este trabalho investigou a dissolução de potássio em uma solução de ácido cítrico $2 \%$ ( $\mathrm{m} / \mathrm{m}$ ) após prévio processamento térmico e químico, com aditivos $\left(\mathrm{CaCO}_{3}, \mathrm{MgCl}_{2} .6 \mathrm{H}_{2} \mathrm{O}\right)$, da rocha contendo glauconita, em seis temperaturas: $660^{\circ} \mathrm{C}, 700^{\circ} \mathrm{C}, 740^{\circ} \mathrm{C}, 780^{\circ} \mathrm{C}, 820^{\circ} \mathrm{C}$ e $860^{\circ} \mathrm{C}, 60$ minutos e granulometria menor que $0,15 \mathrm{~mm}$. A relação mássica (rocha, $\left.\mathrm{CaCO}_{3}, \mathrm{MgCl}_{2} \cdot 6 \mathrm{H}_{2} \mathrm{O}\right)$ foi: $(1,0: 0,2: 0,3)$. Para este objetivo, foi selecionada uma rocha contendo glauconita da região de Cedro do Abaeté - MG, teor de $11,9 \%$ de $\mathrm{K}_{2} \mathrm{O}$ e $58 \%$ de glauconita. A avaliação da extração de potássio foi por extração em solução de ácido cítrico $2 \%(\mathrm{~m} / \mathrm{m})$, temperatura de $95^{\circ} \mathrm{C}$, durante 15 minutos. A maior extração de potássio foi de $78 \%$ na temperatura de $770^{\circ} \mathrm{C}$. A curva passa por um máximo $\left(770^{\circ} \mathrm{C}\right)$, mostrando que há um limite para a temperatura no processamento térmico. Acima deste valor há uma queda na extração indicando mecanismo diferente de reação entre a rocha e aditivos. Considerando o intervalo até $770^{\circ} \mathrm{C}$, o processamento térmico torna o minério do Verdete uma potencial fonte alternativa de potássio para produção de fertilizante de liberação lenta.

Palavras-Chave: Potássio; Verdete; Processamento Térmico; Glauconita.

Abstract: Glauconite $\left(\mathrm{K}_{2}(\mathrm{MgFe})_{2} \mathrm{Al}_{6}\left(\mathrm{Si}_{4} \mathrm{O}_{10}\right)_{3}(\mathrm{OH})_{12}\right)$ is a monoclinic mineral, greenish, phyllosilicate hydrated of potassium and iron. It is an alternative source of potassium. This work investigated the dissolution of potassium in a solution of $2 \mathrm{wt} \%$ citric acid after previous thermal and chemical processing with additives $\left(\mathrm{CaCO}_{3}, \mathrm{MgCl}_{2} \cdot 6 \mathrm{H}_{2} \mathrm{O}\right)$ of glauconita-bearing rock at six temperatures: $660^{\circ} \mathrm{C}, 700^{\circ} \mathrm{C}, 740^{\circ} \mathrm{C}, 780^{\circ} \mathrm{C}, 820^{\circ} \mathrm{C}$ and $860^{\circ} \mathrm{C}, 60$ minutes and particle size less than $0.15 \mathrm{~mm}$. The mass ratio rock: $\mathrm{CaCO}_{3}: \mathrm{MgCl}_{2} \cdot 6 \mathrm{H}_{2} \mathrm{O}$ was: 1.0:0.2:0.3. For this purpose, a rock containing glauconite from the Cedro do $\mathrm{Abaeté}$ $\mathrm{MG}$, with $11.9 \%$ of $\mathrm{K}_{2} \mathrm{O}$ and ( $58 \%$ of glauconita) was selected. The evaluation of potassium extraction was by carried out in citric acid 2 wt $\%$ solution at $95^{\circ} \mathrm{C}$ for 15 minutes. The highest extraction of potassium (K) was $78 \%$ at the temperature of $770^{\circ} \mathrm{C}$. The curve of extraction goes through a maximum $\left(770^{\circ} \mathrm{C}\right)$, showing that there is a limit to the temperature in the thermal processing. Above this value there is a drop in the extraction indicating different mechanisms of reaction between the rock and additives. Considering the range up to $770^{\circ} \mathrm{C}$, thermal processing makes Verdete ore a potential alternative source of potassium for the production of long-term fertilizer.

Keywords: Potassium; Verdete; Thermal Processing; Glauconite.

\section{INTRODUCÃO}

Dentre as rochas portadoras de potássio com baixa disponibilidade para as plantas o verdete se destaca pelo seu teor de $\mathrm{K}_{2} \mathrm{O}$, que varia entre $6 \%$ e $15 \%$. Sua coloração verde característica se deve à existência de íons $\mathrm{Fe}_{2}+$ na estrutura da glauconita, o seu principal constituinte mineral (Piza, et al., 2011), um silicato lamelar hidratado de potássio e ferro. O verdete apresenta, além da glauconita em sua composição, minerais potássicos de baixa solubilidade em água, como a ilita, biotita e muscovita. Essa rocha apresenta outros nutrientes como cálcio, magnésio, manganês, ferro e o silício. É encontrado predominantemente na região do AltoParanaíba, Estado de Minas Gerais (Silva et al., 2012). As pesquisas geológicas na região da Serra da Saudade tiveram início na década de 1960, como foco na prospecção de fosfato em Cedro do Abaeté. Com os resultados positivos, O DNPM tornou-se o responsável pela pesquisa geológica. De acordo com Vallareli et al. (1993), na região de Cedro do Abaeté a reserva de verdetes foi calculada em 1,5 bilhões de toneladas a $11 \%$ de $\mathrm{K}_{2} \mathrm{O}$. A região está inserida na Bacia do São Francisco e compreende rochas do Grupo Bambuí (Ackroyd et al., 2014). O Grupo Bambuí é a unidade estratigráfica mais importante, de maior extensão e que contém as mineralizações de potássio.
Os siltitos verdes (verdete) ocorrem associados à formação Serra da Saudade, perfazendo cerca de $70 \mathrm{~m}$ de espessura e são muito homogêneos, tanto em termos texturais e mineralógicos, quanto na distribuição de potássio.

Os fertilizantes de liberação lenta (long-term fertilizer) do potássio usam, principalmente, filossilicatos como matéria-prima provedora do potássio, devido à baixa mobilidade deste elemento em água, ácidos diluídos ou ácidos orgânicos fracos. Para aumentar a cinética de liberação do potássio, várias pesquisas neste sentido têm sido publicadas (Tokunaga, 1991; Vallareli, 1993; Piza et al, 2011, Orioli Jr \& Coutinho, 2009; Mangrich et al, 2001). Resultados obtidos indicam que rochas contendo glauconita podem constituir fontes alternativas de K para uso agrícola. Entre os anos de 1970 a 1980 as pesquisas foram incrementadas, e biotita, leucita, sienito nefelínico, micaxisto, feldspato potássico, cloritaxisto, muscovita e verdete foram alguns dos materiais avaliados (Lopes et al. 1972; Faquin, 1982; Leite, 1985). A prospecção, caracterização geoquímica, definição de métodos de processamento e avaliação agronômica sistemática dessas rochas resultam na identificação de fontes alternativas eficientes do nutriente, dando origem a um novo grupo de insumos agrícolas (Resende et al, 2006).

Uma maneira de aumentar a disponibilidade do potássio no solo é promover uma modificação na estrutura 
mineral por processamento térmico (Francisco et al, 2007; Silva et al, 2012; Van Straaten, 2007). Mazumder et al (1993) investigaram a dissolução de potássio de glauconita em água destilada, após processamento térmico entre $700^{\circ} \mathrm{C}$ e $850^{\circ} \mathrm{C}$ usando uma mistura de arenito, contendo o mineral, e cloreto de cálcio, variando o tempo de calcinação entre 5 e 180 minutos e granulometria de $300 \mu \mathrm{m}$ a $75 \mu \mathrm{m}$. A lixiviação em água destilada foi executada em temperaturas que variaram de $40^{\circ} \mathrm{C}$ a $90^{\circ} \mathrm{C}$, conseguindo extrações maiores que $90 \%$ a partir de 10 minutos de lixiviação, a $850^{\circ} \mathrm{C}$. A relação arenito:cloreto de cálcio para atingir estas extrações foi de 1,0:0,5 em um tempo de 60 minutos.

O objetivo do presente trabalho foi o estudo da glauconita como fonte alternativa de potássio para produção de um fertilizante de liberação lenta. Mais propriamente, visou-se avaliar a influência da temperatura no processamento térmico da rocha, com aditivos, na solubilidade do potássio numa solução de ácido cítrico $2 \%(\mathrm{~m} / \mathrm{m})$ e caracterizar os sólidos processados termicamente por difratometria de raios-X.

\section{MATERIAIS E MÉTODOS CARACTERIZAÇÃO QUÍMICA E MINERALÓGICA}

Para caracterização química e mineralógica, as amostras foram cominuídas em granulometrias adequadas às análises. A microscopia eletrônica de varredura (Microscópio Eletrônico de Varredura, marca JEOL, modelo JSM 35C e Espectrômetro de Energia Dispersiva de Raios-X, marca NORAN, modelo Voyager 3050) foi aplicada para identificação das fases minerais e seus elementos.

Para análise dos componentes cristalinos da amostra foi usado difratômetro de raios- $X$, Philips-PANalytical PW 1710 do laboratório de Raios-X do Departamento de Engenharia Metalúrgica e de Materiais da UFMG, com uma voltagem de $40 \mathrm{mV}$, corrente de $20 \mathrm{~mA}$ e ângulo de incidência variando de $3^{\circ}$ a $80^{\circ}$, com variação de $0,05^{\circ}$. Nos difratogramas gerados, os picos de difração foram indexados de acordo com os padrões apresentados no banco de dados ICCD. Para a análise por ICP/OES a preparação da amostra seguiu os procedimentos abaixo:

- Fusão por metaborato de lítio: a fusão envolveu a dissolução completa da amostra em fluxo fundido;

- Espectrometria de Emissão Ótica com Plasma Indutivamente Acoplado Leitura direta - ICP/OES: o equipamento utilizado neste trabalho foi o ICP/OES modelo Perkin Elmer Optima 7300DV.

\section{PROCESSAMENTO TÉRMICO}

O siltito verde investigado é proveniente de rocha (verdete) contendo glauconita que ocorre na Serra da Saudade, na região do município de Cedro de Abaeté MG - Brasil. Para o processamento térmico foi considerado o sistema: rocha: $\mathrm{CaCO}: \mathrm{MgCl} 2.6 \mathrm{H} 2 \mathrm{O}$, na relação mássica 1,0:0,2:0,3.
Levando em consideração resultados da pesquisa de Aitta et al (1986) sobre uma melhor distribuição granulométrica para tratamento térmico de rochas contendo glauconita, foi adotado o tamanho de partículas: $100 \%$ menor que $0,15 \mathrm{~mm}$ para a amostra utilizada no processamento térmico. Para escolha da temperatura, foram conduzidas simulações de equilíbrio de fases considerando os componentes de entrada. As simulações foram feitas no aplicativo FactSage módulo Equilib. Para o sistema rocha: $\mathrm{CaCO}_{3}: \mathrm{MgCl}_{2} \cdot 6 \mathrm{H}_{2} \mathrm{O}$ as fases possíveis de se formarem encontram-se na Tabela 1.

Tabela 1: Fases presentes para o sistema glauconita $/ \mathrm{CaCO}_{3} / \mathrm{MgCl}_{2}$. $6 \mathrm{H}_{2} \mathrm{O}$ obtidas por simulação no FactSage. G-Gás, L-Líquido e S-Sólido.

\begin{tabular}{lccccc}
\hline Temperatura $\left({ }^{\circ} \mathrm{C}\right)$ & 800 & 850 & 900 & 950 & 1000 \\
\hline $\mathrm{CO}_{2}$ & $\mathrm{G}$ & $\mathrm{G}$ & $\mathrm{G}$ & $\mathrm{G}$ & $\mathrm{G}$ \\
\hline $\mathrm{H}_{2} \mathrm{O}$ & $\mathrm{G}$ & $\mathrm{G}$ & $\mathrm{G}$ & $\mathrm{G}$ & $\mathrm{G}$ \\
\hline $\mathrm{HCl}$ & $\mathrm{G}$ & $\mathrm{G}$ & $\mathrm{G}$ & $\mathrm{G}$ & $\mathrm{G}$ \\
\hline $\mathrm{KCl}$ & $\mathrm{L}$ & $\mathrm{L}$ & $\mathrm{L}$ & $\mathrm{G}, \mathrm{L}$ & $\mathrm{G}, \mathrm{L}$ \\
\hline $\mathrm{K}_{2} \mathrm{Cl}_{2}$ & - & - & - & - & $\mathrm{G}$ \\
\hline $\mathrm{Mg}_{2} \mathrm{SiO}_{4}$ & $\mathrm{~S}$ & $\mathrm{~S}$ & $\mathrm{~S}$ & $\mathrm{~S}$ & $\mathrm{~S}$ \\
\hline $\mathrm{KCaCl}_{3}$ & $\mathrm{~S}$ & $\mathrm{~S}$ & $\mathrm{~S}$ & $\mathrm{~S}$ & $\mathrm{~S}$ \\
\hline $\mathrm{CaAl}_{2} \mathrm{Si}_{2} \mathrm{O}_{8}$ & $\mathrm{~S}$ & $\mathrm{~S}$ & $\mathrm{~S}$ & $\mathrm{~S}$ & $\mathrm{~S}$ \\
\hline $\mathrm{MgOCaOSi}_{2} \mathrm{O}_{4}$ & $\mathrm{~S}$ & $\mathrm{~S}$ & $\mathrm{~S}$ & $\mathrm{~S}$ & $\mathrm{~S}$ \\
\hline $\mathrm{MgAl}_{2} \mathrm{O}_{4}$ & $\mathrm{~S}$ & $\mathrm{~S}$ & $\mathrm{~S}$ & $\mathrm{~S}$ & $\mathrm{~S}$ \\
\hline
\end{tabular}

Em função do aparecimento de $\mathrm{KCl}$ nas fases gasosas em temperaturas acima de $950^{\circ} \mathrm{C}$ e que a diminuição da fase sólida $\mathrm{KCaCl}_{3}$ acima de $900^{\circ} \mathrm{C}$, as temperaturas escolhidas foram: $660^{\circ} \mathrm{C}, 700^{\circ} \mathrm{C}, 740^{\circ} \mathrm{C}, 780^{\circ} \mathrm{C}, 820^{\circ} \mathrm{C}$ e $860^{\circ} \mathrm{C}$. A escolha do $\mathrm{CaCO}_{3}$ teve a função de diminuir a perda de $\mathrm{HCl}$ devido à decomposição do $\mathrm{MgCl}_{2} \cdot 6 \mathrm{H}_{2} \mathrm{O}$.

$\mathrm{O}$ processamento térmico foi executado em cadinho cerâmico (alumina) com uma massa inicial de $50 \mathrm{~g}$ de rocha, mantida na temperatura por $1 \mathrm{~h}$ e granulometria menor que $0,15 \mathrm{~mm}$. A relação mássica no sistema de mistura rocha- $\mathrm{CaCO}_{3}-\mathrm{MgCl}_{2} \cdot 6 \mathrm{H}_{2} \mathrm{O}$ foi $1,0: 0,2: 0,3$ e todos os componentes da mistura foram reagentes $\mathrm{PA}$.

\section{RESULTADOS E DISCUSSÃO CARACTERIZAÇÃO QUÍMICA E MINERALÓGICA}

A composição mineralógica da amostra está representada no gráfico da figura 1 . A composição química da amostra de verdete encontra-se na Tabela 2

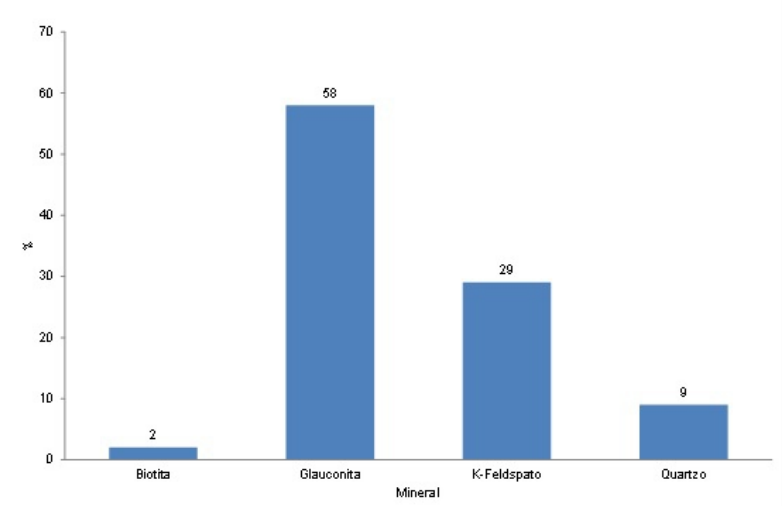

Figura 1: Composição mineralógica da amostra de Verdete usada na investigação. 
Tabela 2 : Composição química da amostra de Verdete usada na investigação.

\begin{tabular}{cccccccc}
\hline Composto & $\mathrm{Al}_{2} \mathrm{O}_{3}$ & $\mathrm{CaO}$ & $\mathrm{Fe}_{2} \mathrm{O}_{3}$ & $\mathrm{~K}_{2} \mathrm{O}$ & $\mathrm{MgO}$ & $\mathrm{Na}_{2} \mathrm{O}$ & $\mathrm{SiO}_{2}$ \\
\hline$\%$ & 15,1 & $<0,03$ & 6,7 & 11,9 & 3,0 & 0,0 & 62,2 \\
\hline
\end{tabular}

A figura 2 representa o difratograma da rocha do verdete com suas principais leituras. As imagens no MEV ( fig. 3) mostram uma matriz predominante de filossilicatos.

As imagens de MEV/EDS mostram um padrão genérico de uma matriz de filossilicatos. Nos difratogramas de raios- $X$ há a superposição dos picos da illita com os picos da glauconita (já que a estrutura é micácea). Não foi, portanto, possível identificar a glauconita somente com estes métodos isolados. Em função disto, a afirmação da presença da glauconita baseou-se na caracterização prévia de minério da região executada por Moreira (2015), que a partir de um estudo mineralógico detalhado identificou a glauconita como o sendo o principal mineral portador de potássio, atingindo até $80 \%$ destes filossilicatos.

\section{PROCESSAMENTOTÉRMICO}

O difratograma de uma mistura tratada termicamente a $780^{\circ} \mathrm{C}$ por 60 minutos está apresentado na figura 4 . As fases cristalinas identificadas são: D - Diopsídio ( $\mathrm{C}$ a $\mathrm{Mg} \mathrm{Si}_{2} \mathrm{O}_{6}$ ), $\mathrm{M}-\mathrm{GI}$ a u con i t a $\left(\mathrm{K}_{2}(\mathrm{MgFe})_{2} \mathrm{Al}_{6}\left(\mathrm{Si}_{4} \mathrm{O} 10\right)_{3}(\mathrm{OH})_{12}\right)$, O-Ortoclásio (KAISi3O8), $\mathrm{Q}$ - Quartzo $\left(\mathrm{SiO}_{2}\right)$, S - Silvita $(\mathrm{KCl})$. A silvita foi prova contundente do deslocamento do potássio da estrutura da glauconita. A $780^{\circ} \mathrm{C}$ a mica residual é pouco identificada, indicando a transformação quase total desta fase, lembrando que o limite de detecção de uma análise por DRX é de 5\%. O aparecimento do diopsídio, fase rica em Ca e $\mathrm{Mg}$, é mais uma evidência de reação representada pelo modelo de núcleo não reagido com formação de camada de cinzas, que no presente sistema é a fase inerte (diopsídio).

\section{EXTRAÇÃO DOS SISTEMAS PROCESSADOS TERMICAMENTE EM ÁCIDO CÍTRICO}

O gráfico da figura 5 mostra a extração de potássio $(K)$ em ácido cítrico, seguindo metodologia do MAPA (MAPA, 2017), após ensaios da mistura (com aditivos) em várias temperaturas. A curva passa por um máximo, em torno de $770^{\circ} \mathrm{C}$, mostrando que há um limite para a temperatura. Acima deste valor há uma queda na extração, indicando que outras reações secundárias e irreversíveis provavelmente ocorrem.

\section{CONCLUSÃO}

$\mathrm{O}$ processamento térmico da mistura (verdete, $\mathrm{CaCO}_{3}$, $\mathrm{MgCl}_{2} \cdot 6 \mathrm{H}_{2} \mathrm{O}$ ) na proporção mássica de 1,0:0,2;0,3 foi efetiva no aumento da solubilização do potássio contido, resultando em $78 \%$ de extração deste elemento em uma solução de ácido cítrico $2 \%(\mathrm{~m} / \mathrm{m})$. A formação de silvita (KCl) foi observada, comprovando o deslocamento do potássio da estrutura dos filossilicatos. A extração aumenta com a temperatura, passando por um máximo $(78 \%)$ a $770^{\circ} \mathrm{C}$. Acima deste valor a extração de potássio diminui. Acima desta temperatura outras reações podem ocorrer, diminuindo a solubilidade do potássio em ácido cítrico e consequentemente sua liberação no solo.

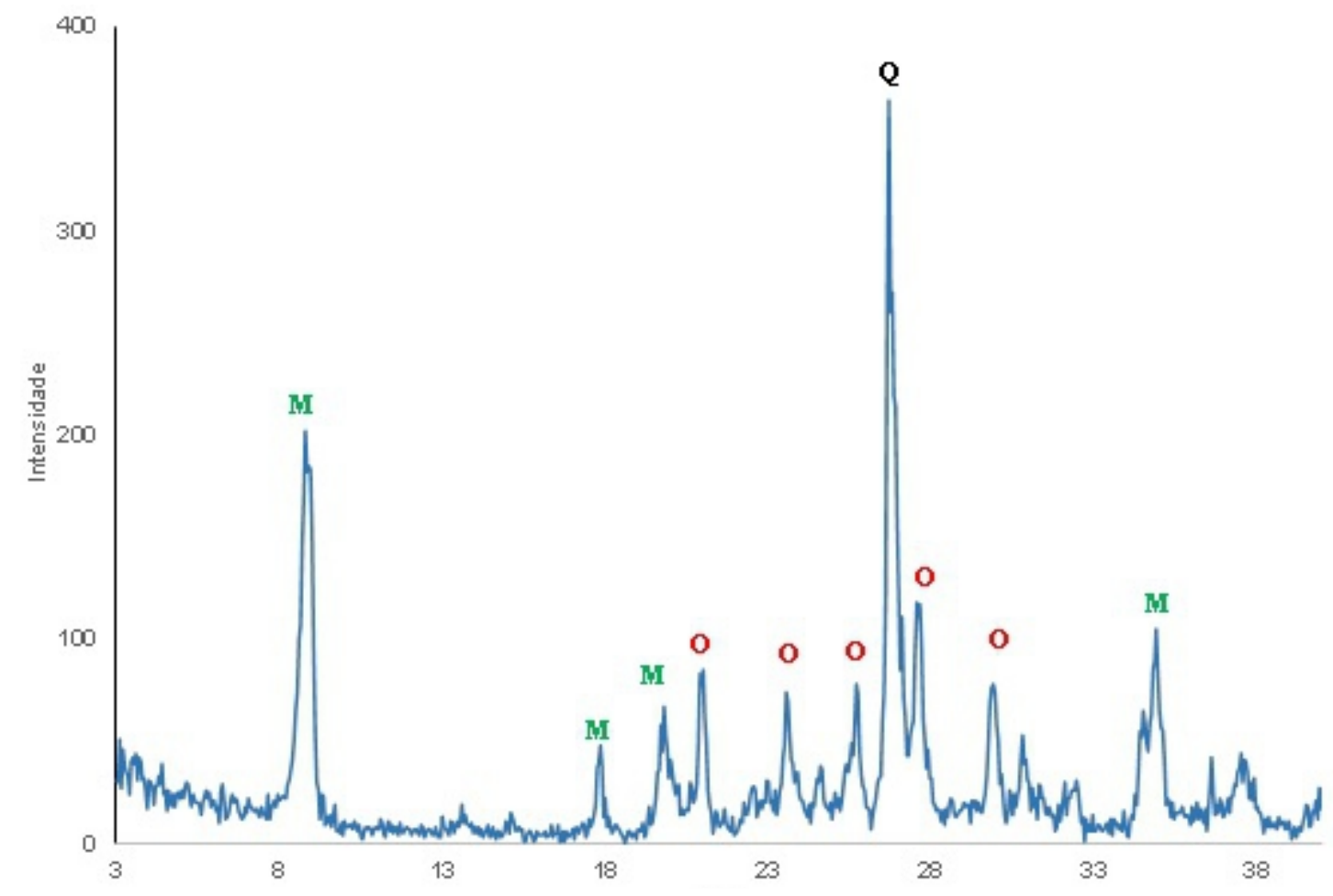

Figura 2-Difratograma do minério verdete: M-Glauconita, Q-Quartzo, O-Ortoclásio (K-Feldspato). 

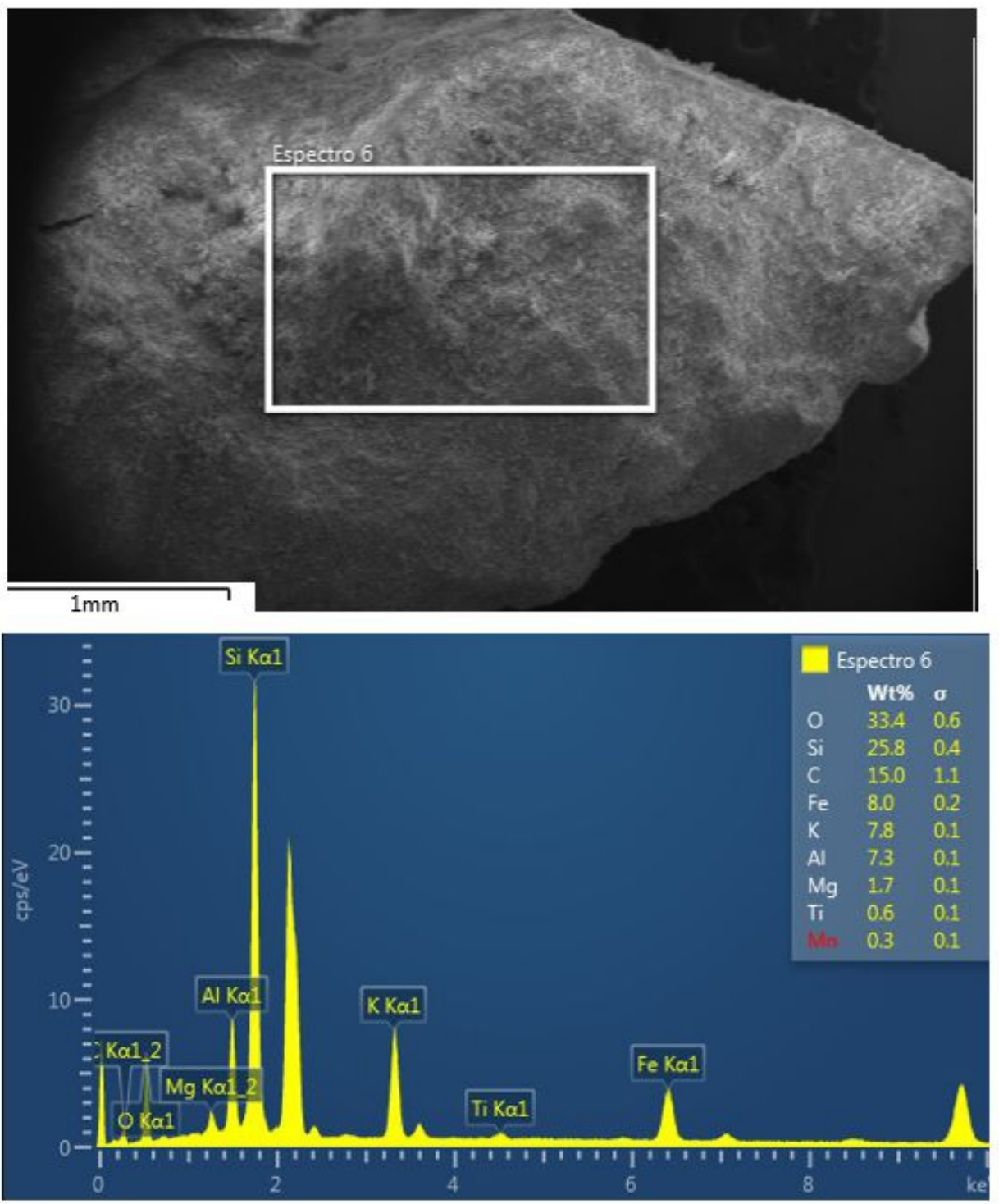

Figura 3-Imagens de elétrons retroespalhados de amostra de rocha de Verdete em MEV mostrando o predomínio de uma matriz de filossilicatos.

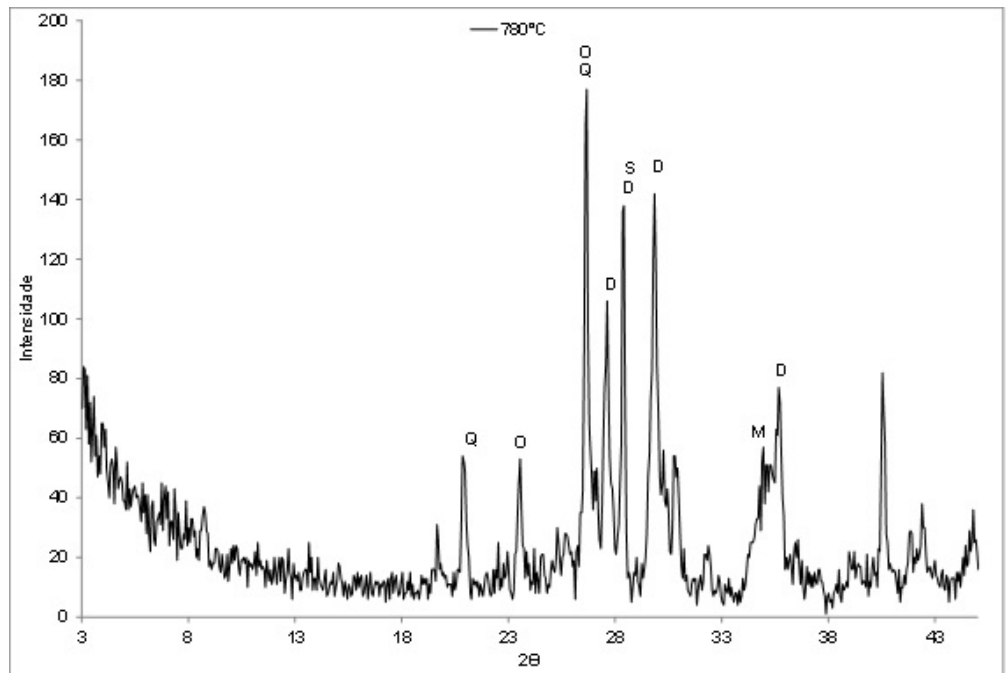

Figura 4 - Difratograma da amostra (mistura) sujeita ao tratamento térmico a $780^{\circ} \mathrm{C}$. Fases identificadas: D - Diopsídio; M - Glauconita; O - Ortoclásio; Q Quartzo; S-Silvita.. 


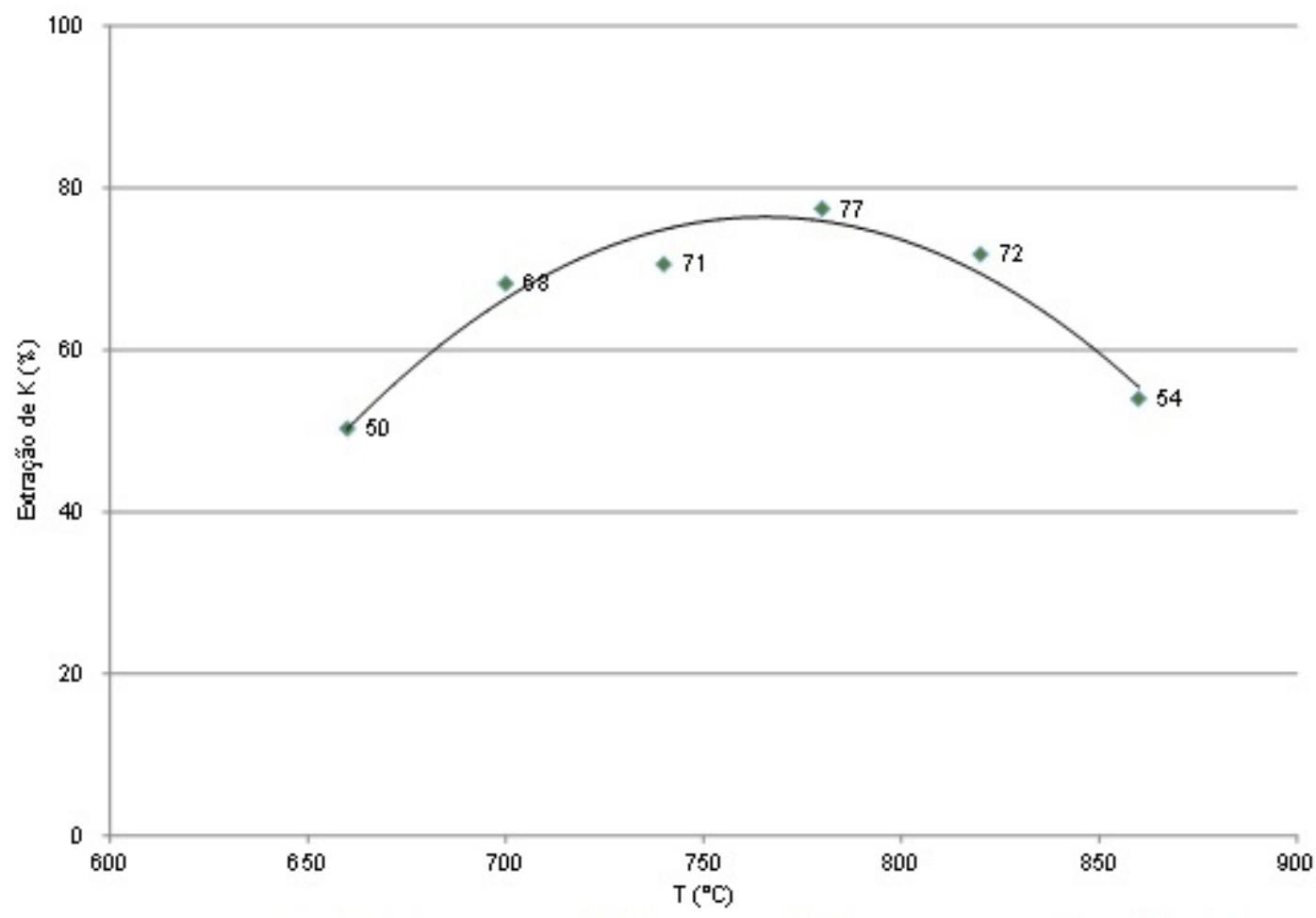

Figura 5-Extração de potássio em solução de ácido cítrico 2\% (m/m).

\section{REFERÊNCIAS BIBLIOGRÁFICAS}

Ackroyd, B; Guzman, C; Chow, W. Pre-Feasibility Study, Cerrado Verde ThermoPotash Project, Minas Gerais State, Brazil. NI 43-101. Technical Report. 260p. 2014.

Aitta, E., Leskela, M., Lajunen, L.H.J., Jyrkas, K., Seppala, E. Thermal Processing /of Phlogopite and Muscovite with Calcium and Magnesium Compounds. Journal of Chemical Technology and Biotechnology, v.36, p.169$177,1986$.

Brasil. Ministério da Agricultura, Pecuária e Abastecimento. Manual de métodos analíticos oficiais para fertilizantes e corretivos / Ministério da Agricultura, Pecuária e Abastecimento. Secretaria de Defesa Agropecuária.-Brasília : MAPA, 2017. 240 p.

Faquin, V. (1982) Efeito do tratamento térmico do sienito nefelínico adicionado de calcário dolomítico, na disponibilidade de potássio ao milho (Zea mays L.), em casa de vegetação. Dissertação (Mestrado) Escola Superior de Agricultura Luiz de Queiroz, Piracicaba. 115p.

Francisco, E.A.B.; Prochnow, L.I.; Toledo, M C M.; Ferrari, V.C.; Jesus, S.L. Thermal Treatment of Aluminous Phosphates of the Crandallite Group and Its Effect on Phosphorus Solubility. Scientia Agricola, v.64, n.3, p.269-274, 2007.
Leite, P.C. (1985) Efeitos de tratamentos térmicos em misturas de verdete de Abaeté, fosfato de Araxá e calcário magnesiano, na disponibilidade de potássio e fósforo. Dissertação (Mestrado) - Escola Superior de Agricultura de Lavras, Lavras. 146p.

Lopes, A.S.; Freire, J.C.; Aquino, L.H., Felipe, M.P. (1972) Contribuição ao estudo da rocha potássica - Verdete de Abaeté (Glauconita) para fins agrícolas. Agros, 2:3242.

Mangrich, A., Tessaro, L., dos Anjos, A., Wypych, F., Soares, J. A slow-release $\mathrm{K}+$ fertilizer from residues of the Brazilian oil-shale industry: synthesis of kalsilitetype structures. Environmental geology, v.40 n.8 p.1030-1036, 2001.

Mazumder, A.K., Sharma, T., Rao, T.C. Extraction of potassium from glauconitic sandstone by the roastleach method. International Journal of Mineral Processing, v.38, p.111-123, 1993.

Moreira, D.A. Estratigrafia, petrografia e Gênese da Mineralização de Potássio em Siltitos Verdes (Verdetes) do Grupo Bambuí na região de São Gotardo, Minas Gerais. (Dissertação de Mestrado) Instituo de Geociências. Universidade Federal de Minas Gerais. Belo Horizonte. 146p. 2015 
Orioli Jr, V., Coutinho, E.L.M. "Effectiviness of Fused Magnesium Potassium Phosphate for Marandu Grass". Revista Brasileira de Ciência do Solo, v. 33 pp. 1855-1862, 2009.

Piza, P.A.T, Bertolino, L.C., Silva, A.A.S., Sampaio, J.A., Luz, A.B. Verdete da região de Cedro do Abaeté (MG) como fonte alternativa para potássio. Geociências, 30:345-356, 2011.

Resende, A.V., Martins, E.S., Oliveira, C.G., Sena, M.C., Machado, C.T.T., Kinpara, D.I., Oliveira Filho, E.C. Suprimento de potássio e pesquisa de uso de rochas "in natura" na agricultura brasileira. Espaço \& Geografia, v.9, n.1, 2006.

Silva, A.A.S., Medeiros, M.E.; Sampaio, J.A.; Garrido, F.M.S. Caracterização do Verdete de cedro do abaeté para o desenvolvimento de material com liberação controlada de potássio. HOLOS, Ano 28, v.5, 2012.

Tokunaga, Y. Potassium silicate: a slow-release potassium fertilizer. Kaihatsu Hiryo Co.2-1-13. 1991.

Vallareli. J.V. Ardósias Verdete de Cedro do Abaeté na Produção de Termofosfato Potássico Fundido e sua Eficiência Agronômica. Anais da Academia Brasileira de Ciências, v.31, p.363-375, 1993.

Van Straaten, P. Agrogeology: the use of rocks for crops. Ontario (Canadá): Enviroquest Ltd,. 2007, 426p. 